\title{
A 53-nW 9.1-ENOB 1-kS/s SAR ADC in 0.13-m CMOS for Medical Implant Devices
}

Dai Zhang, Ameya Bhide and Atila Alvandpour

\section{Linköping University Post Print}

N.B.: When citing this work, cite the original article.

(C2012 IEEE. Personal use of this material is permitted. However, permission to reprint/republish this material for advertising or promotional purposes or for creating new collective works for resale or redistribution to servers or lists, or to reuse any copyrighted component of this work in other works must be obtained from the IEEE.

Dai Zhang, Ameya Bhide and Atila Alvandpour, A 53-nW 9.1-ENOB 1-kS/s SAR ADC in 0.13-m CMOS for Medical Implant Devices, 2012, IEEE Journal of Solid-State Circuits, (47), 7, 1585-1593.

http://dx.doi.org/10.1109/JSSC.2012.2191209

Postprint available at: Linköping University Electronic Press

http://urn.kb.se/resolve?urn=urn:nbn:se:liu:diva-80792 
Paper title: A 53-nW 9.1-ENOB 1-kS/s SAR ADC in $0.13-\mu \mathrm{m}$ CMOS for Medical Implant Devices

Authors: Dai Zhang, Ameya Bhide, and Atila Alvandpour

Affiliation: Department of Electrical Engineering, Linköping University, Linköping, Sweden Authors' contact information:

Dai Zhang

Student Member, IEEE

Email: dai@isy.liu.se

Tel: +46(0)13-282703
Ameya Bhide

Student Member, IEEE

Email: ameya@isy.liu.se

Tel: +46(0)13-288946
Atila Alvandpour

Senior Member, IEEE

Email: atila@isy.liu.se

Tel: +46(0)13-285818

\section{Abstract}

This paper describes an ultra-low power SAR ADC for medical implant devices. To achieve the nano-watt range power consumption, an ultra-low power design strategy has been utilized, imposing maximum simplicity on the ADC architecture, low transistor count and matched capacitive DAC with a switching scheme which results in full-range sampling without switch bootstrapping and extra reset voltage. Furthermore, a dual-supply voltage scheme allows the SAR logic to operate at $0.4 \mathrm{~V}$, reducing the overall power consumption of the ADC by $15 \%$ without any loss in performance. The ADC was fabricated in $0.13-\mu \mathrm{m}$ CMOS. In dual-supply mode $(1.0 \mathrm{~V}$ for analog and $0.4 \mathrm{~V}$ for digital), the ADC consumes $53 \mathrm{nW}$ at a sampling rate of $1 \mathrm{kS} / \mathrm{s}$ and achieves the ENOB of 9.1 bits. The leakage power constitutes $25 \%$ of the 53-nW total power.

Index Terms - ADC, analog-to-digital conversion, low-power electronics, successive approximation, leakage power consumption, medical implant devices. 


\section{INTRODUCTION}

Medical implant devices, such as pacemakers and implantable cardiac defibrillators, target increasingly advanced signal acquisition and signal processing systems. Such devices, which are to be implanted in the human body, require extremely low power consumption in order to operate up to 10 years or more [1]. Analog-to-digital converters (ADCs) are among the most critical and power hungry components of medical implant devices for measurements of various electrophysiological signals (DC to a few $\mathrm{kHz}[2])$. Conversion of the low-frequency analog signals does not need high speed, but requires ultra-low-power operation (e.g. in nW range). This combined with the required conversion accuracy makes the design of such ADCs a major challenge. So far, most of the research on ADCs has been focused on moderate and particularly high-speed applications, while efficient design methodologies and circuit techniques for lowspeed and ultra-low-power ADCs have not been explored in depth.

This paper describes a 10-bit 1-kS/s successive approximation register (SAR) ADC in 0.13- $\mu \mathrm{m}$ CMOS for medical implant devices [3]. Trading speed for lower power at such slow sampling rates is not a straightforward task. The major challenge is how to efficiently reduce the unnecessary speed and bandwidth for ultra-low-power operation using inherently fast devices in advanced CMOS technologies. Moreover, the leakage currents contribute to a significant portion of the total power consumption. As an example, 0 shows the average power consumption of an inverter in a $0.13-\mu \mathrm{m}$ CMOS technology as a function of its switching frequency. The power consumption was simulated at two different supplies $(1.0 \mathrm{~V}$ and $0.4 \mathrm{~V})$ over two different sizes $\left(W_{\min } / L_{\min }\right.$ and $\left.W_{\min } / 2 L_{\min }\right)$. It can be seen that the leakage power at $1-10 \mathrm{kHz}$ can constitute more than $50 \%(50 \%$ at $10 \mathrm{kHz})$ of the total power. 


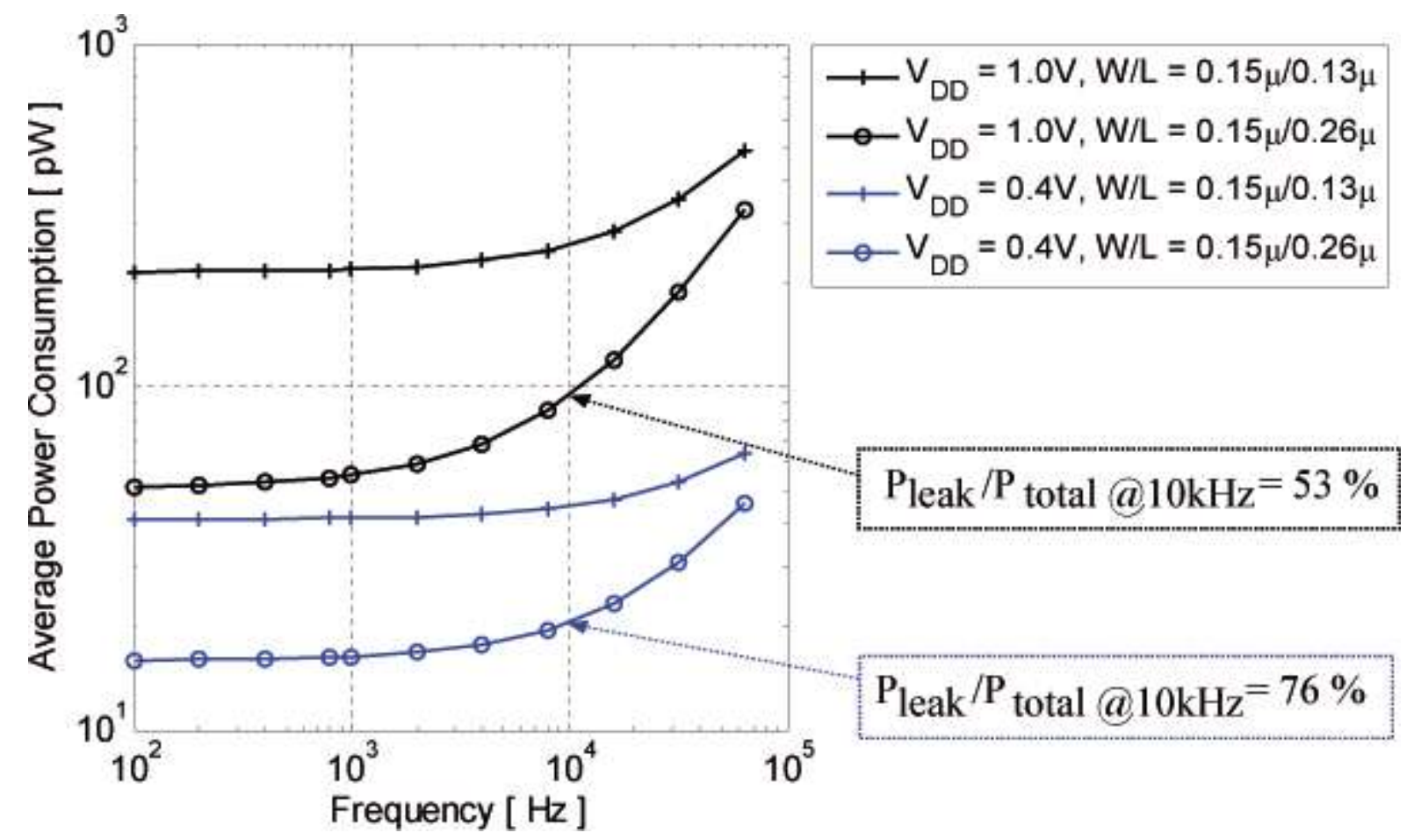

Fig.1: Simulated average power consumption versus switching frequency of an inverter with a fan-out of four in $0.13-\mu \mathrm{m}$ CMOS.

Considering the above discussion and the fact that every nano-watt counts for such ADCs, the main key to achieve the ultra-low-power operation turns out to be the maximal simplicity in the ADC architecture and low transistor count. This essentially means that we avoid ADC techniques with additional complexity and circuit overhead, which are useful for higher sampling rates. Digital error correction [4]-[6] has been frequently used in high-speed ADCs, where capacitor redundancy is utilized to meet the linearity requirement without degrading the speed. However, the circuit overhead required for the digital post-processing leads to additional switching and leakage power consumption. On-chip digital calibration [7][8] serves as an alternative solution without large amount of digital post-processing, but it requires additional calibrating capacitor arrays and registers. Besides, to ensure the calibration efficiency, the comparator offset should be removed prior to linearity calibration. Taking advantage of the low $3(24)$ 
speed, the proposed ADC utilizes a matched capacitive DAC, being sized to achieve the 10-bit conversion accuracy without digital error correction or calibration, thus eliminating additional devices and significant leakage currents. Moreover, the matched capacitive DAC uses a switching scheme that allows full-range sampling without switch bootstrapping and extra reset voltages. Compared to the energy-efficient switching schemes [9]-[11], the employed approach introduces less overhead in the SAR control logic [9][10] and avoids additional bias voltages in the comparator [11]. To further reduce the power consumption, a dual-supply voltage scheme was employed, allowing the SAR logic to operate at $0.4 \mathrm{~V}$. Utilizing the above design strategy combined with low-leakage circuit techniques, careful circuit optimizations, and circuit layout, the SAR ADC consumes $53 \mathrm{nW}$ power at a sampling rate of $1 \mathrm{kS} / \mathrm{s}$, achieving $9.1 \mathrm{ENOB}$.

The paper is organized as follows. Section II describes the ADC architecture. Section III presents the detailed circuit design of the ADC. The measurement results and comparison with previous works are shown in section IV, followed by conclusions in section V.

\section{ADC ARCHITECTURE}

0 shows the block diagram of the proposed ADC. It comprises a matched binary-weighted capacitive DAC, a low-power dynamic latch comparator, a low-leakage/low-voltage synchronous SAR digital logic, and level shifters between the digital logic and the analog blocks. In addition, a differential architecture was employed to have a good common-mode noise rejection.

In a conventional SAR ADC [12], the input voltage is sampled on the bottom-plate nodes of the capacitor array and the top-plate nodes are reset with a fixed voltage. The fixed voltage is commonly chosen to be one of the power rails in order to avoid extra voltage levels. However, 


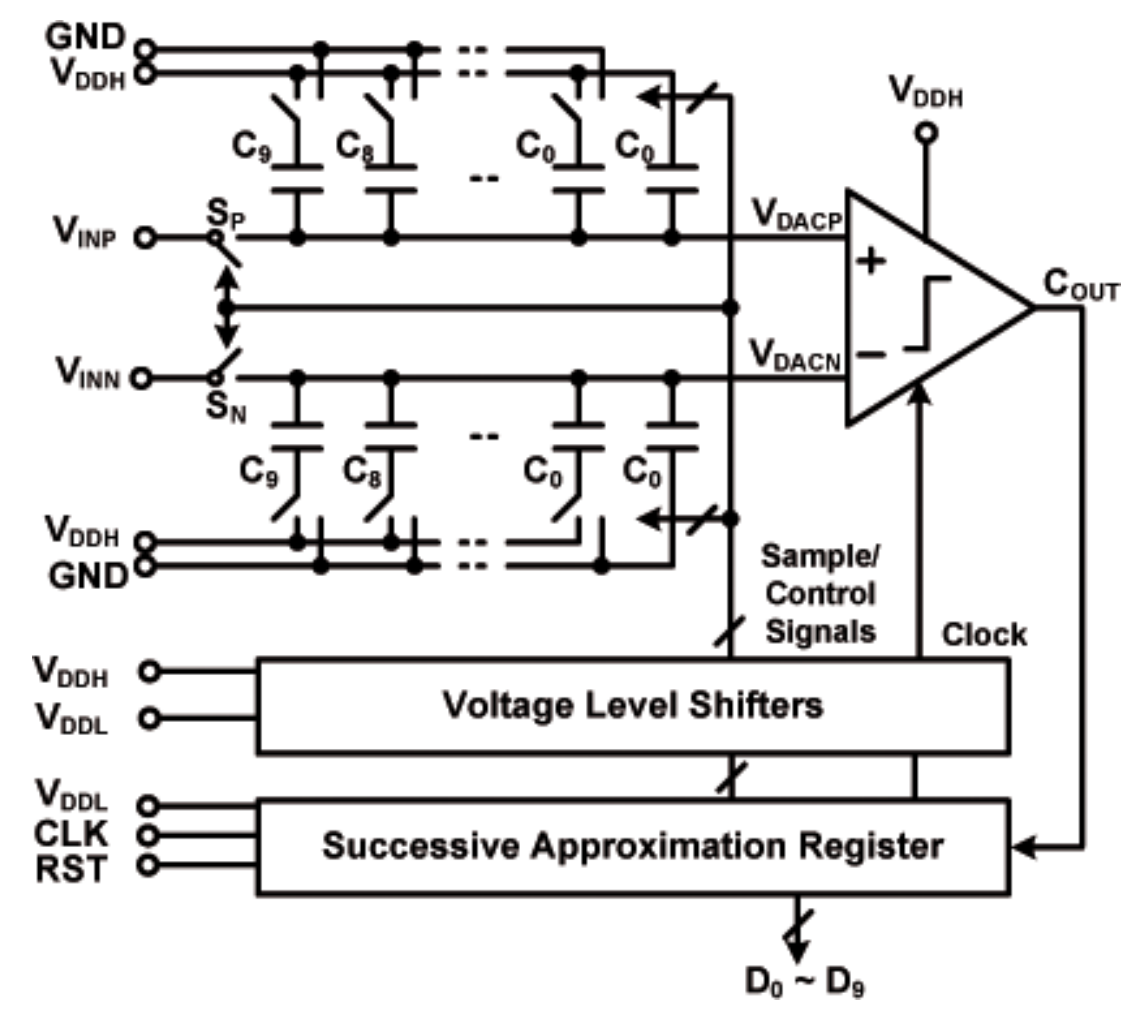

Fig. 2: Architecture of the SAR ADC.

this makes the DAC outputs go beyond the rails during the conversion when full-range input sampling is applied. One common way to solve this problem is to decrease the input range with the penalty of degrading the signal-to-noise ratio. Another alternative is to make the top-plate switches bootstrapped. In this work, we use top-plate sampling [10] with MSB preset to achieve full-range sampling without switch bootstrapping and extra reset voltages. As shown in 0 , the differential inputs are initially connected to the top-plates of the capacitor array, and simultaneously the MSB is reset to high and all other bits are reset to low. Next, the top-plate sampling switch is open and the input data is sampled on the capacitor array. The comparator then performs the first comparison. If $V_{D A C P}$ is higher than $V_{D A C N}$, the MSB remains high. Otherwise, it goes low. Then, the second approximation step starts by setting MSB-1 to high, and 


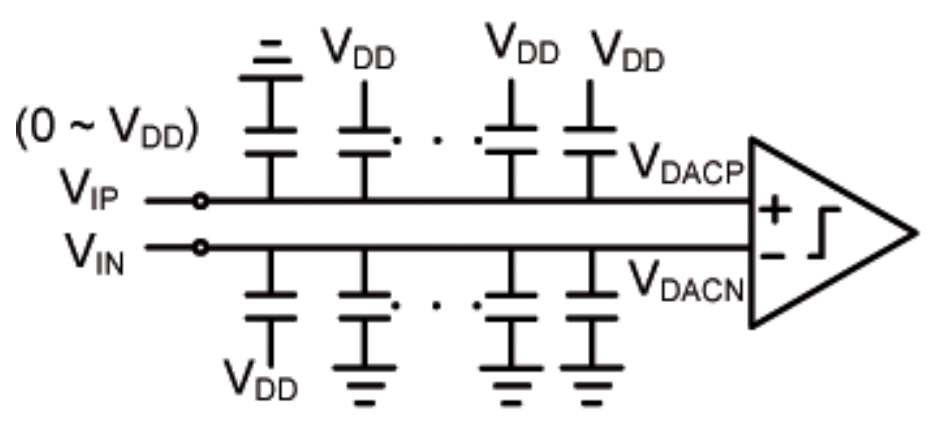

Sampling Phase

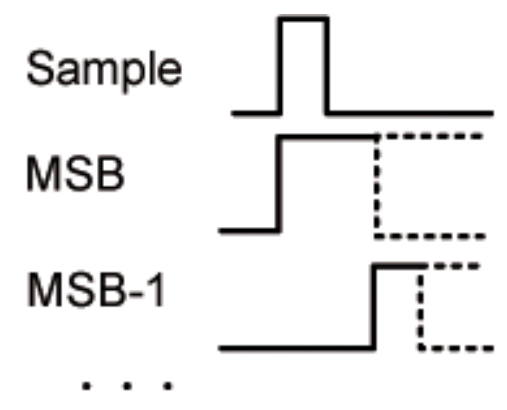

Related Time Sequence

Fig. 3: The sampling phase of capacitive DAC with MSB preset.

the comparator does the comparison again. The ADC repeats this procedure until all 10 bits are decided. During the entire conversion, the DAC outputs always remain within the rails. Moreover, the common-mode voltage of the DAC outputs is the same as that of the differential inputs, which is equal to mid-rail voltage for full-range input sampling, as shown in 0 . The constant common-mode voltage reduces the signal-dependent dynamic offset of the comparator [11].

Lowering the supply voltage is an efficient technique to reduce both the switching and leakage power consumption. This is particularly true at low data-rates, where transistors can be slow but still meet the target speed. However, for the analog circuits operating with low supply voltages, noise and a reduced dynamic range can degrade the ADC performance. To avoid the analog performance degradation, in this design, we use a dual-supply voltage scheme, which allows the SAR logic to operate at low supply voltages. Our measurement results (in Sec. IV) show that this voltage scaling has reduced the overall power consumption of the ADC by $15 \%$ without any loss in performance. 


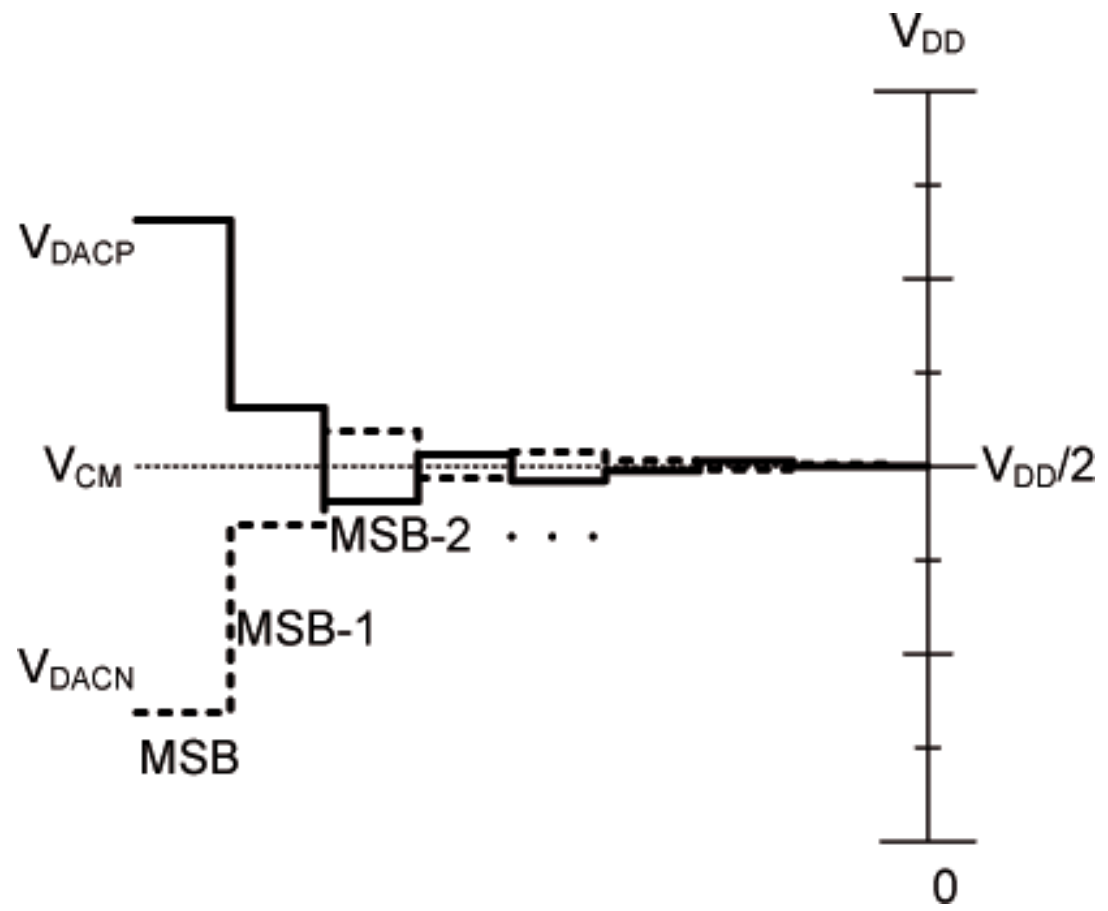

Fig. 4: Waveform of the DAC switching procedure.

\section{CiRCUit ImPLEMENTATION}

In this section, the circuit level design of the DAC, switches, comparator, and SAR digital logic are described. Since these components are critical with regards to power consumption, speed, and accuracy of the entire ADC, much of the design effort was focused on characterizing and optimizing their performance.

\section{A. Capacitive $\mathrm{DAC}$}

The capacitive DAC was implemented with a binary-weighted capacitor array instead of split architecture with an attenuating capacitor [13]-[15]. The split architecture is commonly used to reduce the total number of capacitors. However, this technique is sensitive to mismatch as well as to parasitic capacitance. In addition, to meet the linearity requirements, the total capacitance 
(and the power consumption) of the split architecture can be comparable to those of a conventional binary-weighted structure [16].

The unit capacitor in the DAC should be kept as small as possible for power saving. In practice, it is usually determined by the thermal noise and capacitor mismatch. In this design, mismatch is dominant over thermal noise. Generally, the unit capacitor is modeled with a nominal value of $C_{u}$ and a standard deviation of $\sigma_{u}$. For a binary-weighted capacitor array, the worst-case standard deviation of differential nonlinearity (DNL) and integral nonlinearity (INL) occur at the MSB code transition due to the accumulation of the capacitor mismatch. Following the analysis in [17], they can be expressed in terms of LSB as

$$
\begin{gathered}
\sigma_{D N L, M A X}=\sqrt{2^{N}-1} \frac{\sigma_{u}}{C_{u}} L S B, \\
\sigma_{I N L, M A X}=\sqrt{2^{N-1}} \frac{\sigma_{u}}{C_{u}} L S B,
\end{gathered}
$$

where $N$ is the ADC resolution. Comparing (1) with (2), the derived worst-case standard deviation of DNL is larger than that of INL. Therefore, (1) is chosen to be a reference in the following analysis. For a typical metal-insulator-metal (MIM) capacitor, it has

$$
\sigma\left(\frac{\Delta C}{C}\right)=\frac{K_{\sigma}}{\sqrt{A}}, \text { and } C=K_{C} \cdot A
$$

where $\sigma(\Delta C / C)$ is the standard deviation of capacitor mismatch, $K_{\sigma}$ is the matching coefficient, $A$ is the capacitor area, and $K_{C}$ is the capacitor density parameter. The standard deviation of a single capacitor to the nominal value is by factor $\sqrt{2}$ smaller than that of the difference between two capacitors. Thus, $\sigma(\Delta C / C)$ divided by $\sqrt{2}$ is equal to $\sigma_{u} / C_{u}$. For high yield, it is necessary to 
maintain $3 \sigma_{D N L, M A X}<1 / 2 \mathrm{LSB}$. Combining the earlier equations, we obtain a lower bound for the mismatch-limited unit capacitor

$$
C_{U}=18 \cdot\left(2^{N}-1\right) \cdot K_{\sigma}^{2} \cdot K_{C .}
$$

In this technology, a MIM capacitor has a density of $2 \mathrm{fF} / \mu \mathrm{m}^{2}$ and a matching of $1 \% \mu \mathrm{m}$. It leads to a minimum unit capacitance of $4 \mathrm{fF}$. So far, the discussion is for the single-ended architecture. For the differential configuration in this design, the unit capacitance can be reduced by half while still satisfying the mismatch requirement. This is because the differential mode doubles the signal range but only increases $\sqrt{2}$ times of the voltage error introduced by the mismatch.

Apart from the mismatch, the design rule will also set a minimum value on the MIM capacitance. The minimum MIM capacitance defined by this process is $27 \mathrm{fF}$. Consequently, the unit capacitance was set to be $13.5 \mathrm{fF}$ in our work, which was implemented by two minimum process-defined MIM capacitors in series. Hence, the total array capacitance is about $14 \mathrm{pF}$.

Besides capacitor sizing, a careful layout to avoid linearity degradation is important as well. In this work, we have utilized a partial common-centroid layout strategy for the capacitor array. 0 illustrates the layout floor plan. The MSB capacitors $\left(\mathrm{C}_{9}-\mathrm{C}_{5}\right)$ follow a common-centroid configuration to minimize the errors from the non-uniform oxide growth in the MIM capacitors. However, the smaller LSB capacitors $\left(\mathrm{C}_{4}-\mathrm{C}_{0}\right)$ have been placed close to the bottom-plate switches to simplify the routing, thereby reducing the parasitic capacitances and resistances of the interconnection. Post-layout simulations showed that the reduced parasitic of the employed partial common-centroid layout results in better DAC linearity, compared to a capacitor array with a full common-centroid layout (where the LSB capacitors were placed in the middle of the array). Based on the simulations, the DAC with the partial common-centroid layout had a peak 9 (24) 
DNL of $+0.18 /-0.20$ LSB and INL of $+0.30 /-0.23 \mathrm{LSB}$, while the DAC with a full commoncentroid layout had a peak DNL of +0.35/-0.16 LSB and INL of +0.40/-0.36 LSB.

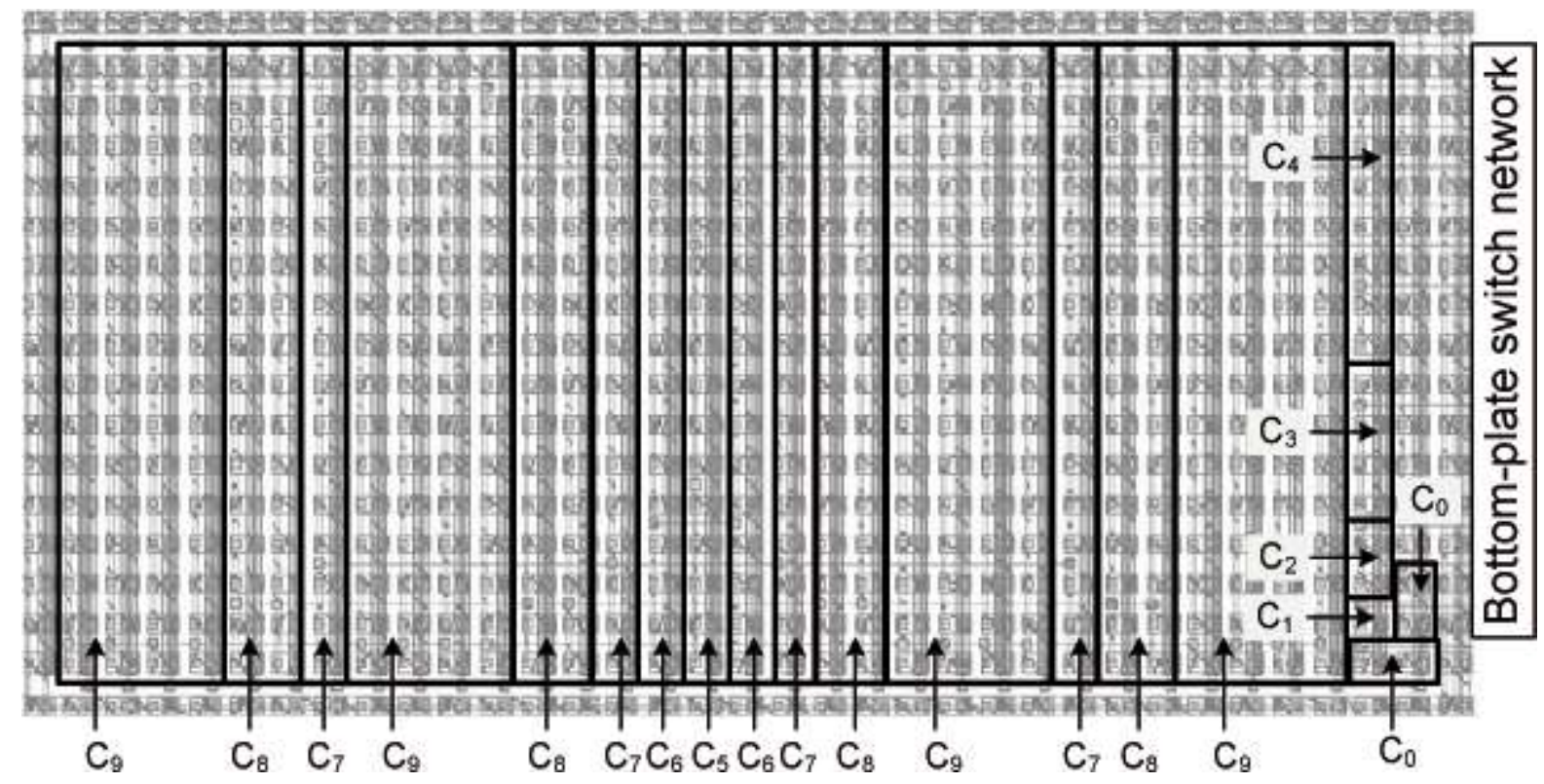

Fig. 5: Layout of the capacitor array which follows a partial common-centroid configuration. The capacitors are indicated according to 0 .

\section{B. Switch Design}

The top-plate sampling switch was implemented using transmission gate, shown in 0 , to achieve full-range input sampling. The switch together with the DAC capacitor array acts as the sample-and-hold circuit of the ADC. If the sampling circuit is designed for an $N$-bit performance, then the settling error of the sampled voltage must be less than half of LSB. This requires [18]

$$
f_{3 d B}>\frac{(N+1) \cdot \ln 2}{\pi} f_{S},
$$

where $f_{S}$ is the sampling frequency. In this design, the sampling time is determined by the system clock, which is $N+2$ times the sampling rate. Hence, (5) can be modified to

$$
f_{3 d B}>\frac{(N+1) \cdot(N+2) \cdot \ln 2}{\pi} f_{S} .
$$




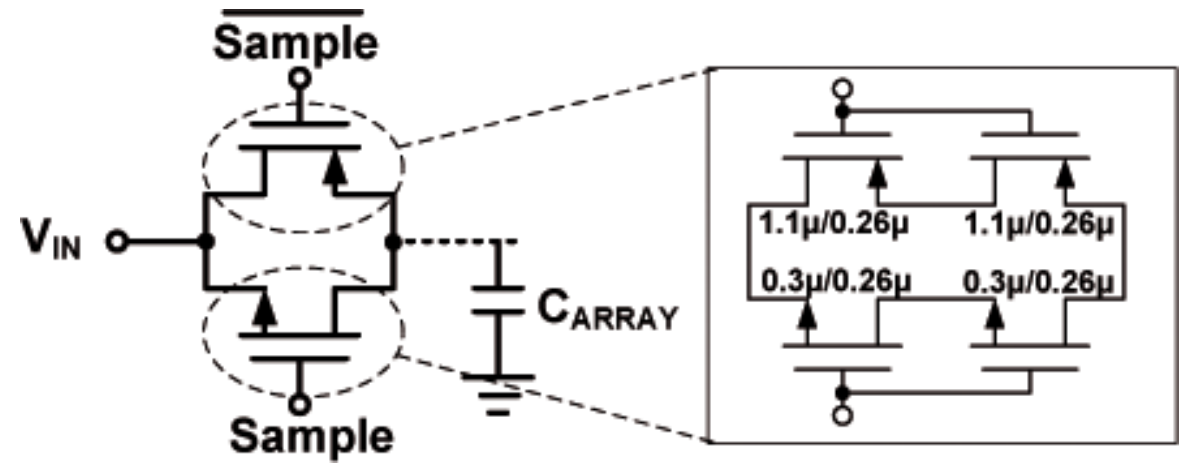

Fig. 6: Top-plate sampling switch.

Based on (6), for a 10-bit $1-\mathrm{kS} / \mathrm{s}$ SAR ADC, the required minimum $f_{3 d B}$ is about $30 \mathrm{kHz}$. Taking account of the 14-pF sampling capacitance, the switch on-resistance $\left(R_{O N}\right)$ should be designed to be less than $380 \mathrm{k} \Omega$.

Apart from the bandwidth requirement, the voltage drop introduced by the leakage current of the switch can also degrade the conversion accuracy due to the low sampling rate. The subthreshold leakage current of the transistor is the dominant leakage contributor to the switch. In addition, the leakage current shows nonlinear dependence on the input-output voltage difference across the switch, thus introducing harmonic distortion. Increasing the channel length is an effective solution to reduce the sub-threshold leakage current. To further reduce the leakage current, we have utilized a two-transistor stack [19] (shown in 0). 0 shows the simulated subthreshold leakage currents of two different switches versus their input voltages. The figure compares the leakage current of a single transistor with a channel length of $4 L_{\min }$ to two transistors in series with channel lengths of $2 L_{\min }$. It can be seen that the stacked transistors show lower leakage for small input voltages in the range from $0 \mathrm{~V}$ to $0.1 \mathrm{~V}$. 


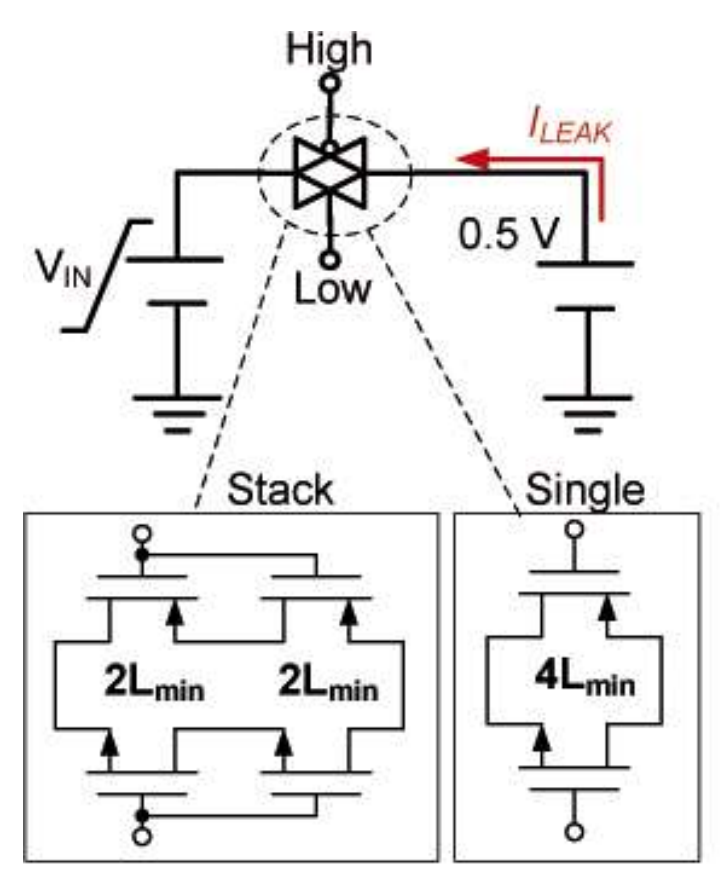

(a)

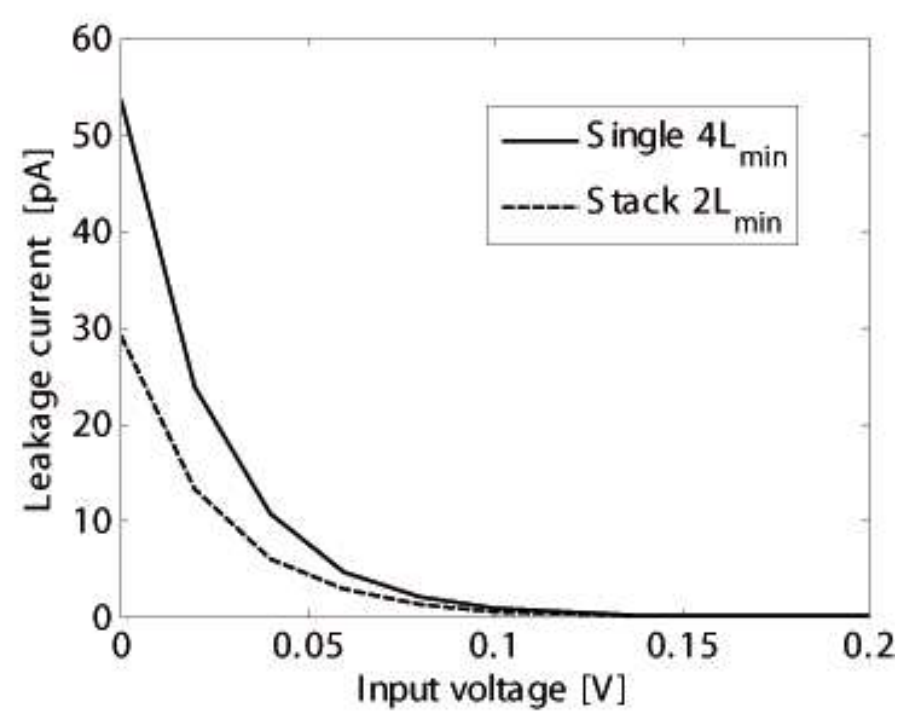

(b)

Fig. 7: Simulated leakage current of the sampling switch: (a) test-bench (b) leakage current versus input voltage

To determine the channel length of the stacked transistors, the sampling circuit was simulated at $1-\mathrm{kHz}$ sampling frequency with full-range input signal for three different switch lengths $\left(L_{\min }+L_{\min }, 2 L_{\min }+2 L_{\min }\right.$, and $\left.3 L_{\min }+3 L_{\min }\right)$. The frequency of the input signal was swept from near-DC to near-Nyquist bandwidth. The voltage of the sampled output signal was recorded at the end of the hold phase to track the voltage drop. The simulated worst-case signal-to-noiseand-distortion ratio (SNDR) of the recorded voltage was found at near-Nyquist operation. The results of SNDR for three different switch lengths were $60.9 \mathrm{~dB}, 67.4 \mathrm{~dB}$, and $67.5 \mathrm{~dB}$, respectively. Thus increasing the total channel length beyond $4 L_{\min }\left(2 L_{\min }+2 L_{\min }\right)$ did not introduce much benefit for the leakage reduction. Hence, in this work, the channel length of the stacked transistors was chosen to be $2 L_{\min }(0.26 \mu \mathrm{m})$. 
Furthermore, we sized the transistor width and simulated the switch $R_{O N}$ over the entire input range under $-40^{\circ} \mathrm{C}$ at the slow process corner. The simulated maximum $R_{O N}$ based on the chosen transistor widths $(0.3 \mu \mathrm{m}$ for NMOS and $1.1 \mu \mathrm{m}$ for PMOS) is about $80 \mathrm{k} \Omega$. With the total array capacitance of $14 \mathrm{pF}$, the $f_{3 d B}$ of the sampling circuit is then calculated to be about $140 \mathrm{kHz}$, which gives a design margin with more than four times the required minimum $f_{3 d B}$.

At the bottom-plate sides, inverters connect the capacitors to the power rails. Ideally, minimum-size transistors can be used for all the inverters because of the low sampling rate, thus minimizing power consumption. In practice, however, special care must be taken during sizing of the MSB inverter. The NMOS top-plate sampling switch introduces parasitic PN-junction on the top-plate node. After an input voltage close to ground is sampled, during the MSB to MSB-1 transition, the voltage on the top-plate node can undershoot below ground, forward-biasing the PN-junction and causing charge loss. To avoid the undershoot voltage, the PMOS transistor in the MSB inverter was sized up to six times the minimum width.

\section{Dynamic Latch Comparator}

The dynamic latch comparator [20] is shown in 0. Buffers have been used to make the output loading identical, followed by an SR latch which stores the comparison result for the entire clock cycle.

Since the input common-mode voltage of the comparator is kept at mid-rail voltage, the total comparator offset appears as static offset, which does not affect the linearity of the ADC [11]. The fundamental limitation on the achievable comparator resolution is thermal noise, which has the usual form as $k T / C$ [21][22]. The post-layout extracted capacitor at the comparator output is 
about $10 \mathrm{fF}$, which makes the thermal noise of the comparator in the same order as the quantization noise of the ADC [10].

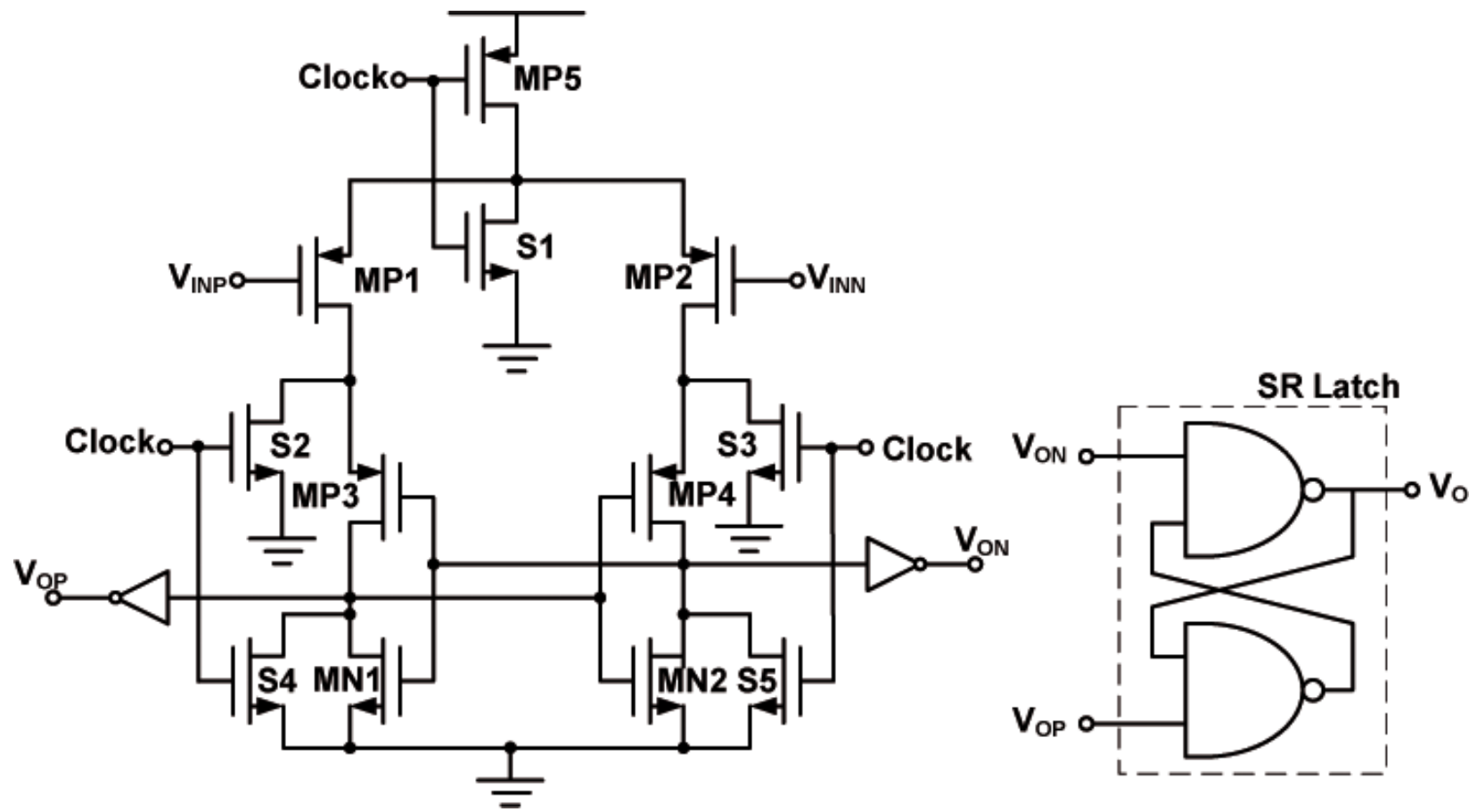

Fig. 8: Dynamic latch comparator [20] and its succeeding SR latch

Though the offset of the comparator does not affect the accuracy, it will decrease the input voltage range, thus degrading the signal-to-noise ratio. Monte Carlo simulations of the comparator offset showed a mean offset of $7.8 \mathrm{mV}$ and a standard deviation of $9.1 \mathrm{mV}$. Assuming $3 \sigma$ is considered, it results in a total offset voltage of $35.1 \mathrm{mV}$, decreasing the SNR by $0.31 \mathrm{~dB}$. The decreased SNR introduces a loss of 0.05-ENOB, which is acceptable in this work.

\section{SAR Control Logic}

For low power SAR control logic, we investigated both synchronous and asynchronous solutions. Asynchronous processing [23] has been frequently used for high-speed SAR ADCs in order to avoid a high-frequency system clock. The SAR control logic starts the conversion on the $14(24)$ 
rising edge of a sampling clock, and triggers the internal comparison from MSB to LSB successively. The delay, usually generated by an inverter line [10], has a large dependency on process, voltage, and temperature variations, which makes it difficult to ensure the DAC settling. Moreover, the short-circuit currents caused by the slow transition of the inverters introduce extra power [10].

The proposed ADC utilizes a synchronous SAR logic, shown in 0. It generates the sample signal and the switch control signals for the DAC. The operation of its multiple-input 10-bit shift resister is similar to [24]. A 4-bit counter and a decoder generate the control signals for the 10-bit shift register. The entire logic uses 16 transmission-gate flip-flops and the decoder has been optimized for minimum logic depth and gate count. 0 shows the time sequence of the SAR logic. A $12-\mathrm{kHz}$ system clock has been used, and the sampling clock of $1 \mathrm{kHz}$ is generated by the SAR logic.

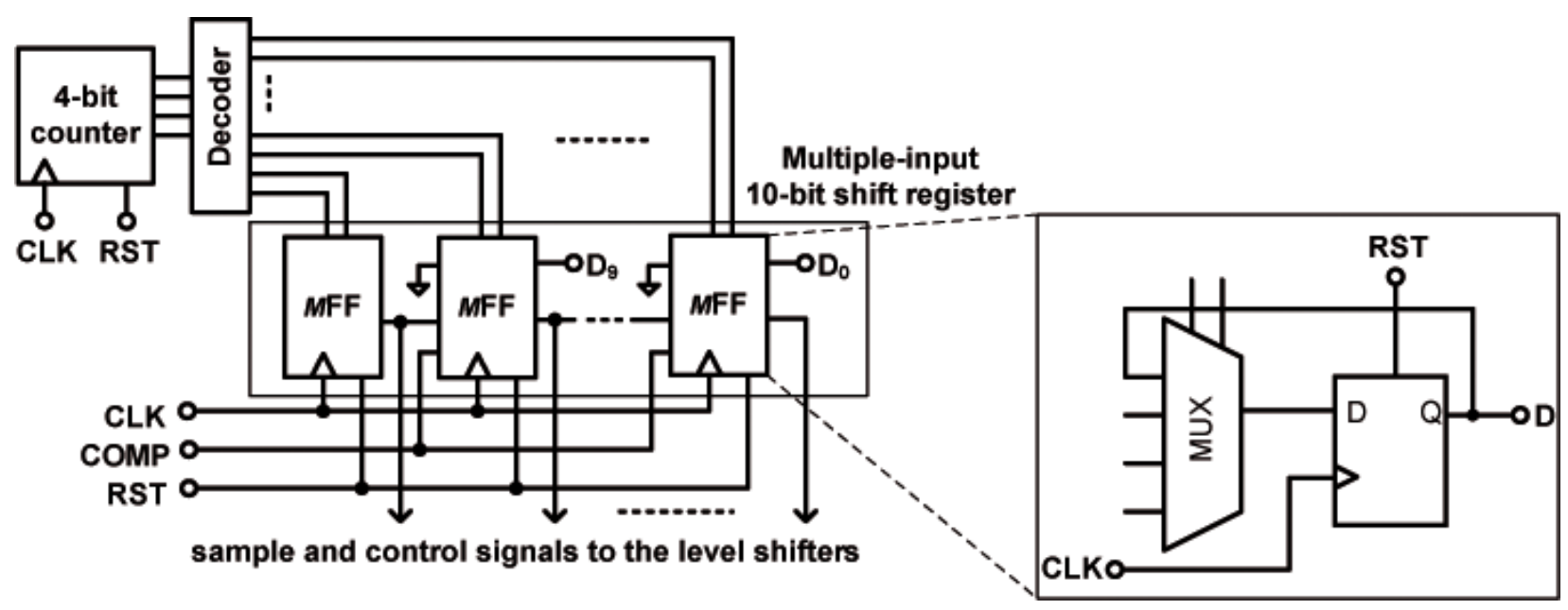

Fig. 9: SAR logic. 


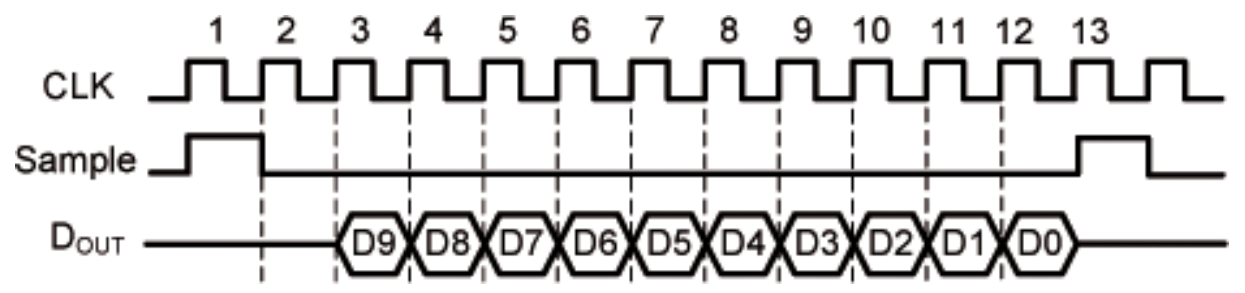

Fig. 10: Time sequence of the synchronous SAR control logic.

Since the operating frequency of the SAR logic is $12 \mathrm{kHz}$, and its switching activity is not high, the leakage power dominates the total power. Several techniques have been used to reduce the leakage currents, including increased channel length, minimum transistor width, and replacing the gate transistors with stacked pairs [25].

To further reduce the switching and leakage power consumption, a dual-supply voltage scheme has been employed, allowing the SAR logic to operate at $0.4 \mathrm{~V}$. The level shifter [26], shown in 0 , has been used to convert the logic levels between the SAR and the analog parts. In the entire ADC, twelve level shifters have been used: ten for the DAC control signals, one for the sampling signal of the top-plate switch, and one for the clock signal of the comparator.

\section{Measurement Results}

The prototype SAR ADC with a core area of $357 \times 536 \mu \mathrm{m}^{2}$ was designed and fabricated in a general purpose $0.13-\mu \mathrm{m}$ one-poly six-metal (1P6M) CMOS process. It was packaged in a $1.27 \mathrm{~mm}$ pitch JLCC package. A photograph of the chip is shown in 0. The unmarked part around the ADC core includes the decoupling capacitors and the I/O buffers for the pads.

Histogram test [27] was conducted to measure the linearity of the ADC. A full-swing, differential sinusoidal input near DC frequency with amplitude of $1 \mathrm{~V}$ was applied to the $1-\mathrm{kS} / \mathrm{s}$ 
ADC. 0 shows the measured DNL and INL error with respect to the output code. The peak DNL error is $+0.54 /-0.61 \mathrm{LSB}$, and the peak INL error is $+0.45 /-0.46 \mathrm{LSB}$.

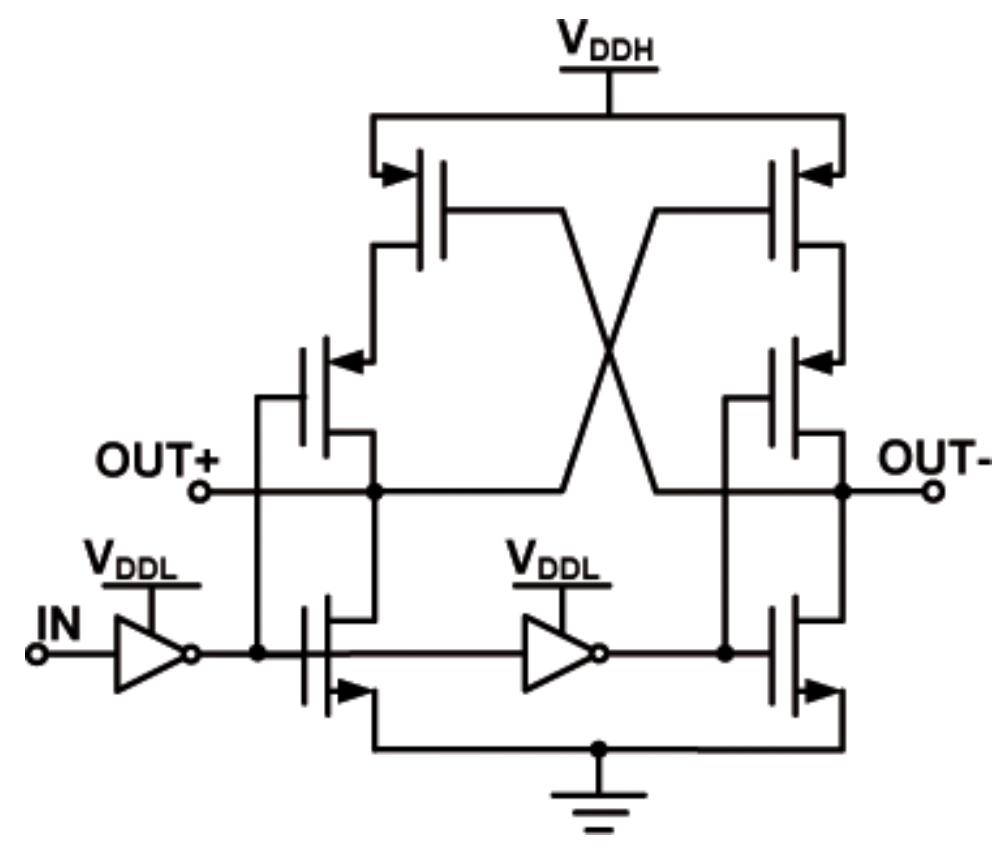

Fig. 11: Level shifter [26].

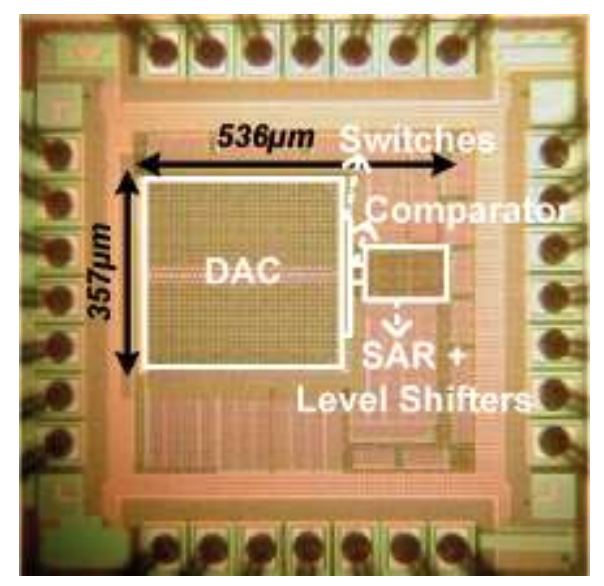

Fig. 12: Die photograph of the ADC in $0.13-\mu \mathrm{m}$ CMOS technology.

The SNDR of the ADC was measured using tone testing. A fast Fourier transform (FFT) of the $1-\mathrm{kS} / \mathrm{s}$ ADC at near-Nyquist operation is shown in 0 . The amplitude of the test stimulus was set to $-0.5 \mathrm{dBFS}$. The measured SNDR is $56.7 \mathrm{~dB}$, providing an effective number of bits (ENOB) 
of 9.1 bits. 0 shows the ENOB of this ADC with respect to the input frequency, where the ENOB remains almost constant over the entire bandwidth. Hence, the effective resolution bandwidth (ERBW) is higher than the Nyquist bandwidth.
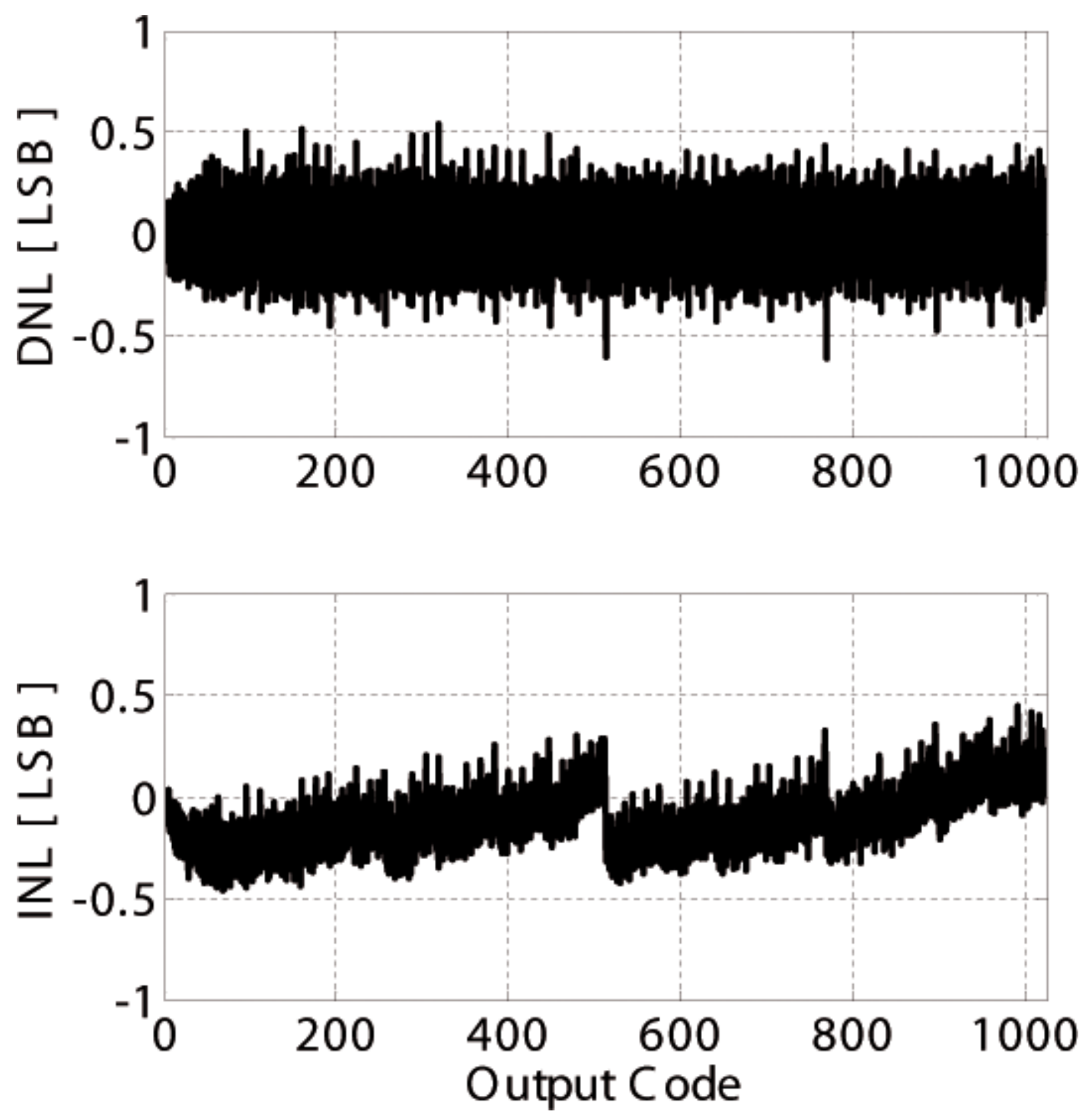

Fig. 13: Measured DNL and INL errors.

Multiple supply voltage domains were utilized, allowing detailed measurement of the power consumption in the DAC, comparator, and SAR control logic, respectively. The total measured power consumption of the $1-\mathrm{kS} / \mathrm{s} \mathrm{ADC}$ is $53 \mathrm{nW}$ in dual-supply mode $\left(V_{D D H}\right.$ of $1.0 \mathrm{~V}$ for DAC 


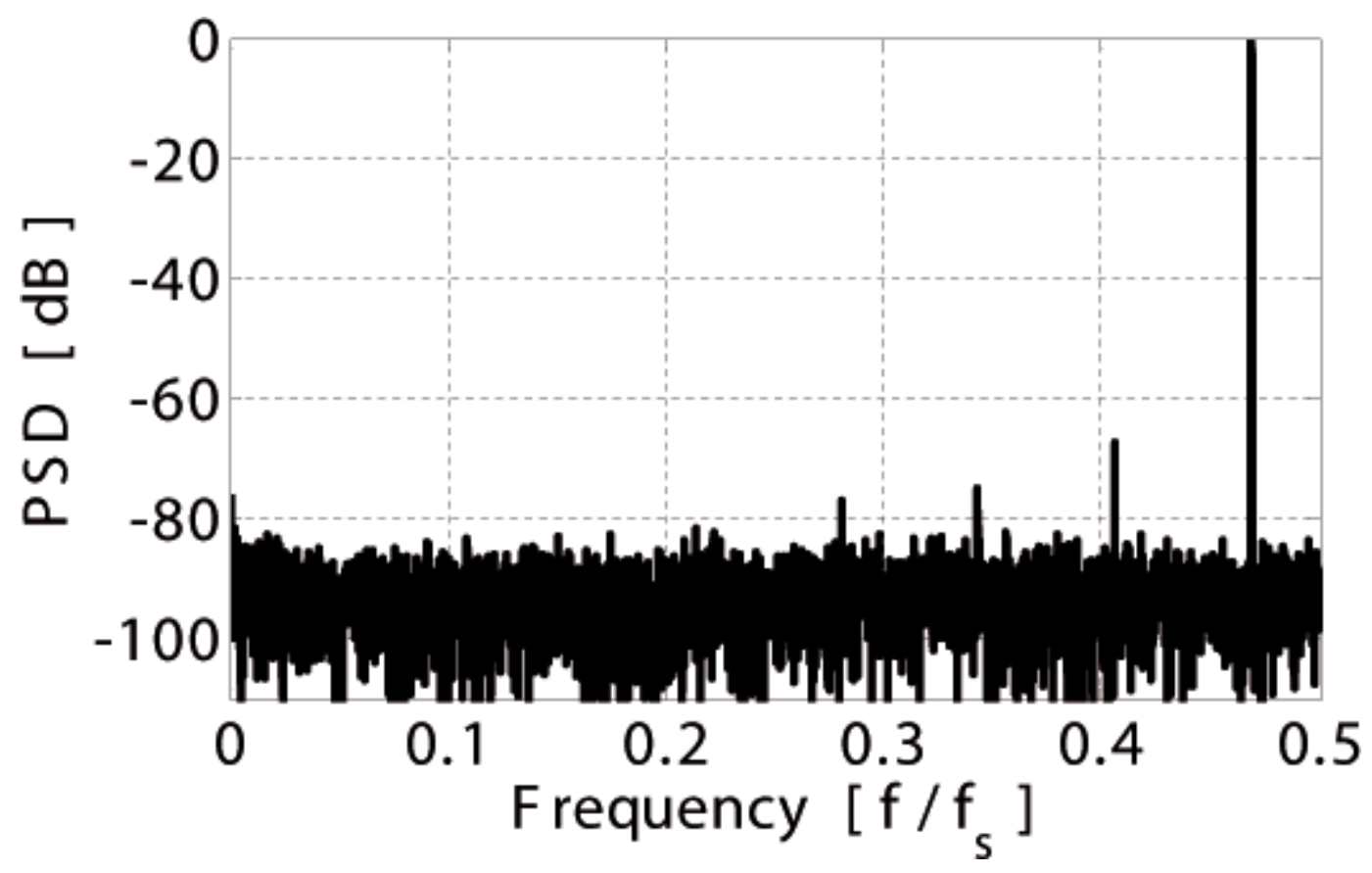

Fig. 14: Measured 8,192-point FFT spectrum at $1 \mathrm{kS} / \mathrm{s}$.

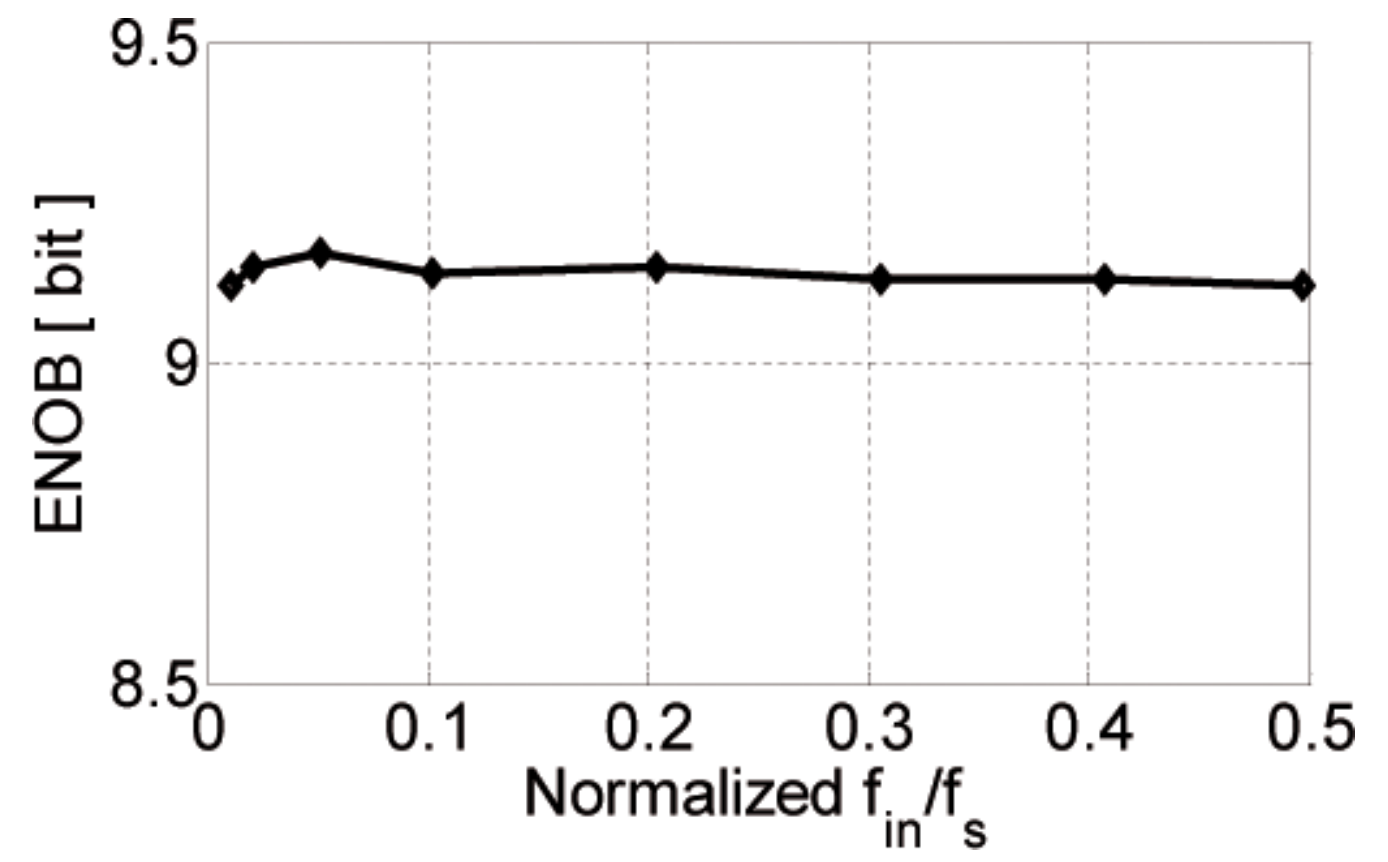

Fig. 15: ENOB of the ADC versus input frequency. 
and comparator, and $V_{D D L}$ of $0.4 \mathrm{~V}$ for SAR logic) and $72 \mathrm{nW}$ in $1-\mathrm{V}$ single-supply mode. The measured leakage power of the digital part is $13 \mathrm{nW}$ in dual-supply mode and $22 \mathrm{nW}$ in $1-\mathrm{V}$ single-supply mode, which constitutes $25 \%$ and $31 \%$ of the total power, respectively. It implies that the digital leakage power consumption is a major contributor to the total power. The power of the level shifters was simulated based on post-layout extraction. In dual-supply mode, the level shifters consume $12 \mathrm{nW}$ power and in $1-\mathrm{V}$ single-supply mode, they consume $10 \mathrm{nW}$ power. Excluding the 10-nW power of level shifters from the total 72-nW power in the singlesupply mode would result in a total ADC power of $62 \mathrm{nW}$. This indicates that the voltage scaling has reduced the overall power consumption of the ADC by $15 \%$ without any loss in performance. 0 shows the power breakdown of the ADC in the two modes. Table I summarizes the measured performance of the ADC.

The power and dynamic performance of the 1-kS/s ADC under different supply settings were measured. The figure-of-merit (FOM) which has been used to compare the ADC performance is defined as

$$
F O M=\frac{\text { Power }}{\min \left\{f_{S}, 2 \times E R B W\right\} \times 2^{E N O B}}
$$

Table II shows the measurement results together with the corresponding FOM. The ADC achieves the optimal performance at $V_{D D H}=1.0 \mathrm{~V}$ and $V_{D D L}=0.4 \mathrm{~V}$ with the lowest FOM of $94.5 \mathrm{fJ} /$ Conversion.

As demonstrated in this work, at such low-sampling rates, leakage power becomes a significant portion of the total power, degrading the FOM as compared to ADCs for higher sampling rates. Therefore, Table III compares the measurement results of this work to previously 
TABLE I ADC MEASUREMENT SUMMARY

\begin{tabular}{|c|c|c|}
\hline \multicolumn{3}{|c|}{ ADC Performance } \\
\hline Technology & \multicolumn{2}{|c|}{$0.13-\mu \mathrm{m} \mathrm{CMOS}$} \\
\hline Sampling Rate & \multicolumn{2}{|c|}{$1 \mathrm{kS} / \mathrm{s}$} \\
\hline Die Area & \multicolumn{2}{|c|}{$357 \times 536 \mu \mathrm{m}^{2}$} \\
\hline DNL & \multicolumn{2}{|c|}{$+0.54 /=0.61 \mathrm{LSB}$} \\
\hline INL & \multicolumn{2}{|c|}{$+0.45 /=0.46 \mathrm{LSB}$} \\
\hline SNDR (at Nyquist) & \multicolumn{2}{|c|}{$56.7 \mathrm{~dB}$} \\
\hline SFDR (at Nyquist) & \multicolumn{2}{|c|}{$67.6 \mathrm{~dB}$} \\
\hline \multicolumn{3}{|c|}{ ADC Power Breakdown } \\
\hline \multirow{3}{*}{$\begin{array}{l}\text { Supply Voltage } \\
\text { Comparator, DAC } \\
\text { SAR }\end{array}$} & & \\
\hline & $1.0 \mathrm{~V}$ & $1.0 \mathrm{~V}$ \\
\hline & $0.4 \mathrm{~V}$ & $1.0 \mathrm{~V}$ \\
\hline Comparator & $2 \mathrm{nW}$ & $2 \mathrm{nW}$ \\
\hline Capacitive DAC & $33 \mathrm{nW}$ & $33 \mathrm{nW}$ \\
\hline Control Logic (SAR + Level Shifter) & $18 \mathrm{nW}$ & $37 \mathrm{nW}$ \\
\hline Total Power & $53 \mathrm{nW}$ & $72 \mathrm{nW}$ \\
\hline
\end{tabular}

TABLE II ADC Performance Under Different Supply Settings

\begin{tabular}{|l|c|c|c|c|}
\hline$V_{D D H} / V_{D C X}[\mathrm{~V}]$ & $0.8 / 0.4$ & $1.0 / 0.4$ & $1.0 / 1.0$ & $1.2 / 1.2$ \\
\hline Power [nW] & 45 & 53 & 72 & 94 \\
\hline ENOB (at Nyquist) [bit] & 8.7 & 9.1 & 9.1 & 9.2 \\
\hline FOM [fJ/Conv.] & 108.1 & 94.5 & 129.4 & 159.8 \\
\hline
\end{tabular}

TABLE III ADC COMPARISON

\begin{tabular}{|l|c|c|c|c|}
\hline $\begin{array}{l}\text { Author/ } \\
\text { Year }\end{array}$ & $\begin{array}{c}\text { Sauerbrey } \\
2003[28]\end{array}$ & $\begin{array}{c}\text { Bechen } \\
2006[29]\end{array}$ & $\begin{array}{c}\text { Zou } \\
2009[30]\end{array}$ & This Work \\
\hline Technology $[\mu \mathrm{m}]$ & 0.18 & 0.8 & 0.35 & 0.13 \\
\hline Sampling Rate $[\mathrm{kS} / \mathrm{s}]$ & 4.1 & 0.8 & $1^{\dagger}$ & 1 \\
\hline Power [nW] & 850 & 3000 & 230 & 53 \\
\hline ENOB [bit] & 6.9 & 9 & 10.2 & 9.1 \\
\hline FOM [fJ/Conv.] & 1700 & 7300 & 195 & 94.5 \\
\hline
\end{tabular}

${ }^{\dagger}$ The sampling rate of $1 \mathrm{kS} / \mathrm{s}$ was reported in [31] and referred in [30] by the same authors. 
published SAR ADCs with comparable sampling rates [28]-[30]. As the table shows, this ADC achieves the lowest power and FOM.

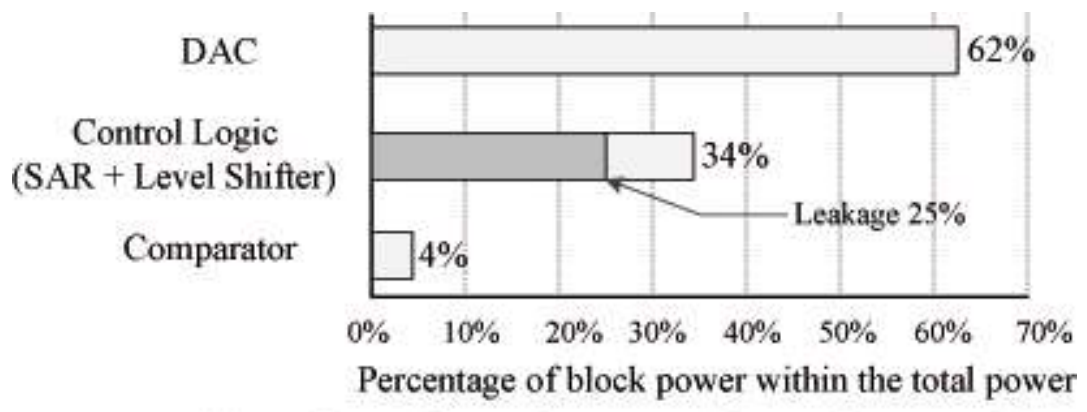

(a) Dual-supply mode $V_{D D H}=1.0 \mathrm{~V}$ and $V_{D D L}=0.4 \mathrm{~V}$

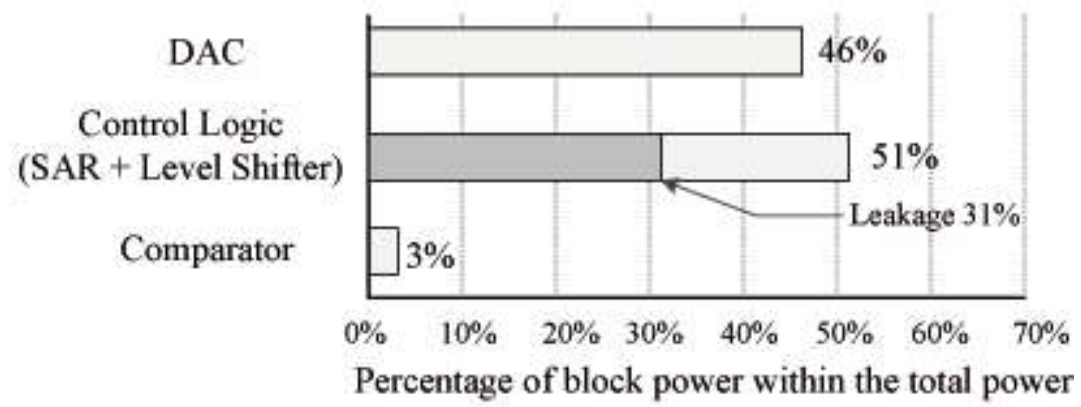

(b) Single-supply mode $V_{D D H}=V_{D D L}=1.0 \mathrm{~V}$

Fig. 16: The ADC power breakdown in dual and single supply modes, where the percentage of digital leakage power is indicated by dark color.

\section{CONCLUSION}

In this paper, we have presented an ultra-low-power SAR ADC in $0.13-\mu \mathrm{m}$ CMOS technology for medical implant devices. The ADC achieves 9.1 ENOB with a power consumption of $53 \mathrm{nW}$ at a sampling rate of $1 \mathrm{kS} / \mathrm{s}$. It utilizes an ultra-low power design strategy, imposing maximum simplicity on ADC architecture, low transistor count, low leakage circuit techniques, and a matched capacitive DAC with a switching scheme which results in full-range sampling without switch bootstrapping and extra reset voltage. Furthermore, a dual-supply scheme allows the SAR logic to operate at $0.4 \mathrm{~V}$, resulting in $15 \%$ power reduction compared to 
the 1-V single-supply mode without any loss in ADC performance. The paper has also shown that at such low-sampling rates, leakage power can be a significant portion of the total ADC power consumption, degrading the energy efficiency and FOM. 


\section{References}

[1]L. S. Y. Wong, S. Hossain, A. Ta, J. Edvinsson, D. H. Rivas, and H. Nääs, "A very lowpower CMOS mixed-signal IC for implantable pacemaker applications," IEEE J. SolidState Circuits, vol. 39, no. 12, pp. 2446-2456, Dec. 2004.

[2] J. G. Webster, Medical Instrumentation Application and Design, Wiley, 1998.

[3] D. Zhang, A. Bhide, and A. Alvandpour, "A 53-nW 9.12-ENOB 1-kS/s SAR ADC in 0.13- $\mu \mathrm{m}$ CMOS for medical implant devices," in Proc. ESSCIRC, pp.467-470, Sep. 2011.

[4]F. Kuttner, "A 1.2V 10b 20MSample/s non-binary successive approximation ADC in 0.13 um CMOS," in IEEE ISSCC Dig. Tech. Papers, pp.176-177, Feb. 2002.

[5] W. Liu, P. Huang, and Y. Chiu, "A 12b 22.5/45MS/s 3.0mW 0.059 mm² CMOS SAR ADC achieving over 90dB SFDR," in IEEE ISSCC Dig. Tech. Papers, pp.380-381, Feb. 2010.

[6] C. C. Liu, S. J. Chang, G. Y. Huang, Y. Z. Lin, C. M. Huang, C. H. Huang, L. Bu, and C. C. Tsai, "A 10b 100MS/s 1.13mW SAR ADC with binary-scaled error compensation," in ISSCC Dig. Tech. Papers, pp.386-387, Feb. 2010.

[7] Y. Chen, X. Zhu, H. Tamura, M. Kibune, Y. Tomita, T. Hamada, M. Yoshioka, K. Ishikawa, T. Takayama, J. Ogawa, S. Tsukamoto, and T. Kuroda, "Split capacitor DAC mismatch calibration in successive approximation ADC, " in Proc. IEEE Custom Integrated Circuit Conf., pp. 279-282, Sep. 2009.

[8] M. Yoshioka, K. Ishikawa, T. Takayama, and S. Tsukamono, "A 10-b 50-MS/s 820- $\mu \mathrm{W}$ SAR ADC with on-chip digital calibration," in IEEE ISSCC Dig. Tech. Papers, pp.384385, Feb. 2010.

[9] B. P. Ginsburg and A. P. Chandrakasan, "500-MS/s 5-bit ADC in 65-nm CMOS with split capacitor array DAC," IEEE J. Solid-State Circuits, vol. 42, no.4, pp. 739-747, April 2007.

[10] M. van Elzakker, E. van Tujil, P. Geraedts, D. Schinkel, E. A. M. Klumperink, and B. Nauta, "A 10-bit charge-redistribution ADC consuming $1.9 \mu \mathrm{W}$ at $1 \mathrm{MS} / \mathrm{s}$," IEEE J. Solid-State Circuits, vol. 45, no.5, pp. 1007-1015, May 2010.

[11] C. C. Liu, S. J. Chang, G. Y. Huang, and Y. Z. Lin, "A 10-bit 50-MS/s SAR ADC with a monotonic capacitor switching procedure," IEEE J. Solid-State Circuits, vol. 45, no.4, pp. 731-740, April 2010.

[12] J. L. McCreary and P. R. Gray, "All-MOS charge-redistribution analog-to-digital conversion techniques-Part I," IEEE J. Solid-State Circuits, vol. SC-10, pp. 371-379, Dec. 1975.

[13] N. Verma and A. P. Chandrakasan, "An ultra low energy 12-bit rate-resolution scalable SAR ADC for wireless sensor node," IEEE J. Solid-State circuits, vol. 42, no. 6, pp. 1196-1205, Jun. 2007.

[14] M. Yip and A. P. Chandrakasan, "A resolution-reconfigurable 5-to-10b 0.4-to-1V power scalable SAR ADC," in IEEE ISSCC Dig. Tech. Papers, pp.190-191, Feb. 2011.

[15] Y. Zhu, C. H. Chan, U. F. Chio, S. W. Sin, S. P. U, R. P. Martins, and F. Maloberti , "A 10-bit 100-MS/s reference-free SAR ADC in $90 \mathrm{~nm}$ CMOS, "IEEE J. Solid-State circuits, vol. 45, no. 6, pp. 1111-1121, Jun. 2010.

[16] M. Saberi, R. Lotfi, K. Mafinezhad, and W. A. Serdijn, "Analysis of power consumption and linearity in capacitive digital-to-analog converters used in successive approximation 
ADCs," IEEE Transactions on Circuits and Systems I: Regular Papers, vol. 58, no. 8, pp. 1736 -1748, Aug. 2011.

[17] T. Wakimoto, H. Li, and K. Murase, "Statistical analysis on the effect of capacitance mismatch in a high-resolution successive-approximation ADC", IEEJ Trans. on Electrical and Electronic Engineering, vol. 6, pp. s89-s93, 2011.

[18] R. Schreier, J. Silva, J. Steensgaard, and G. C. Temes, "Design-oriented estimation of thermal noise in switched-capacitor circuits," IEEE Transactions on Circuits and Systems I: Regular Papers, vol. 52, no. 11, pp. 2358 - 2368, Nov. 2005.

[19] K. Ishida, K. Kanda, A. Tamtrakarn, H. Kawaguchi, and T. Sakurai, "Managing subthreshold leakage in charge-based analog circuits with low- $\mathrm{V}_{\mathrm{TH}}$ transistors by analog T-swtich (AT-Switch) and super cut-off CMOS (SCCMOS)", IEEE J. of Solid-State Circuits, vol. 41, no. 4, pp.859-867, April 2006.

[20] G. Van der Plas and B. Verbruggen, "A 150MS/s 133 $\mu \mathrm{W} 7 \mathrm{~b}$ ADC in 90nm digital CMOS using a comparator-based asynchronous binary-search sub-ADC," in ISSCC Dig. Tech. Papers, pp.242-243, Feb. 2008.

[21] J. Kim, B. Leibowitz, J. Ren. and C. Madden, "Simulation and analysisof random decision errors in clocked comparators," IEEE Transactions on Circuits and Systems I: Regular Papers, vol. 56, no. 8, pp. 1844 -1857, Aug. 2009.

[22] P. Nuzzo, F. De Bernardinis, P.Terreni, and G. Van der Plas, "Noise analysis of regenerative comparators for reconfigurable ADC architecture," IEEE Trans. Circuits Syst. II, vol. 55, no.6, pp. 1441-1454, Jul. 2008.

[23] S. W. M. Chen and R. W. Brodersen, "A 6-bit 600-MS/s 5.3-mW asynchronous ADC in

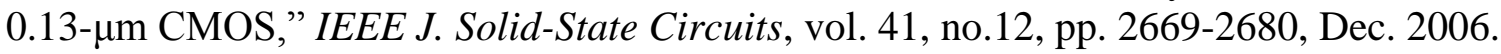

[24] A. Rossi and G. Fucilli, "Nonredundant successive approximation register for A/D converters," Electronics Letters, vol. 32, no. 12, pp. 1055-1056, June 1996.

[25] S. Narendra, V. De, S. Borkar, D. A. Antoniadis, A. P. Chandrakasan, "Full-chip subthreshold leakage power prediction and reduction techniques for sub- $0.18-\mu \mathrm{m}$ CMOS", IEEE J. of Solid-State Circuits, vol. 39, no. 2, pp.501-510, Feb. 2004.

[26] C. Q. Tran, H. Kawaguchi, and T. Sakurai, "Low-power high-speed level shifter design for block-level dynamic voltage scaling environment," in International Conference on Integrated Circuit Design and Technology, pp. 229-232, May 2005.

[27] J. Doernberg, H.-S. Lee, and D. A. Hodges, "Full-speed testing of A/D converters," IEEE J. Solid-State Circuits, vol. SC-19, no.6, pp. 820-827, Dec. 1984.

[28] J. Sauerbery and D. Schmitt-Landsiedel, "A $0.5-\mathrm{V} 1-\mu \mathrm{W}$ successive approximation ADC", IEEE J. of Solid-State Circuits, vol. 38, no. 7, pp.1261-1265, July 2003.

[29] B. Bechen, D. Weiler, T. v. d. Boom, and B.J. Hosticka, "A 10 bit very low-power CMOS SAR-ADC for capacitive micro-mechanical pressure measurement in implants," Advances in Radio Science, Vol. 4, pp. 243-246, 2006.

[30] X. Zou, X. Xu, L. Yao, and Y. Lian, "A 1-V 450-nW fully integrated programmable biomedical sensor interface chip," IEEE J. Solid-State Circuits, vol. 44, no.4, pp. 10671077, April 2009.

[31] X. Xu, X. Zou, L. Yao, and Y. Lian, “A 1-V 450-nW fully integrated biomedical sensor interface system," in Proc. Symposium VLSI Circuits, pp. 78-79, June 2008. 